\title{
Effect of Specimen Geometry on Quantitative EDS Analysis with Four-Quadrant Super-X Detectors
}

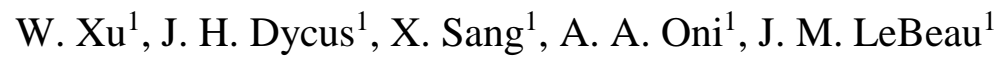 \\ 1. Department of Materials Science and Engineering, North Carolina State University, Raleigh, NC \\ 27695, USA.
}

In recent years, X-ray signal collection efficiency in energy dispersive X-ray spectrometry (EDS) has been significantly improved by incorporating state-of-the-art four-quadrant Super-X detectors in aberration-corrected microscopes $[1,2]$ and has enabled routine atomic-resolution EDS elemental mapping, as shown in Figure 1a. The breakthrough in detector technology has sparked interest in quantifying the chemical information directly from atomically resolved EDS maps [3-5]. In order to obtain an atomic-resolution EDS map, the specimen however must be tilted to a low index zone axis, resulting in different orientation with respect to each detector. This will inevitably result in different Xray signals arriving at each detector due to their different absorption distances and effective take-off angles. Such a variation could largely affect the ability to accurately quantify the overall EDS spectrum from four Super-X detectors without precautions as evidenced in this work. Therefore, understanding the X-ray signal variation from each detector under tilted specimen condition becomes both experimentally and theoretically important as a starting point for atomic-resolution EDS quantification.

In this talk, we will show that the specimen geometry can have a strong effect of on quantitative EDS when using a four-quadrant Super-X detector configuration. $\mathrm{Ni}_{3} \mathrm{Al}$ is an ideal prototype material to investigate geometry effects as the $\mathrm{Ni}$ and $\mathrm{Al}$ characteristic $\mathrm{X}$-ray peaks are well separated and strong absorption of Al-K occurs by $\mathrm{Ni}[6]$. As shown in Figure 1b, a wedge-polished Ni3Al sample was studied using a Titan G2 S/TEM with the sample thin region facing to detectors 3 and 4 in an azimuth angle of 45 degrees. Figure 2a-c show the total intensity of Al-K, Ni-K and Ni-L X-ray signals received from each detector in the same thickness region, respectively. Special care was made to minimize the channeling effect from the specimen. As seen, X-ray peaks obtained from each detector not only vary with the tilt angle, but also systematically shift about 5 degrees towards the positive tilt angle. More importantly, a large deviation of intensity ratios of $\mathrm{Al}-\mathrm{K} / \mathrm{Ni}-\mathrm{K}$ and $\mathrm{Ni}-\mathrm{L} / \mathrm{Ni}-\mathrm{K}$ peaks from different detectors was observed with same specimen tilt as seen in Figure 2d-f. Furthermore, the ratio deviation also varies with the degree of specimen x-tilt, and appears to be non-symmetric, which is correlated with the wedge shape of the specimen. Although the deviation of X-ray signal from four Super-X detectors may be averaged out in the overall spectrum, significant change of the overall intensity ratio occurs at a higher tilt angle even larger than 10-15 degrees. In view of the large quantification uncertainty with the tilt sample, the correction of X-ray signal from overall spectrum for all Super-X detectors is essential and will be discussed. The limitation on tilt angle regarding the absorption, spurious $\mathrm{X}$-ray and fluorescence effect in the Super-X detector configuration will also be presented. The effect of sample shape on the detected X-ray signals from Super-X detectors will be further compared for wedge, FIB and twin-jet polished specimens [7].

\section{References:}

[1] P. Schlossmacher, et al, Microscopy and Analysis (nanotechnology supplement) 24 (2010), p. 55.

[2] L.J. Allen, et al, MRS Bulletin 37 (2012), p. 47. 
[3] G. Kothleitner et al, Physical Review Letters, 112 (2014), p. 085501.

[4] P. Lu et al, Scientific Reports, 4 (2014), p. 3945.

[5] M. Watanabe, Microscopy, 62 (2013), p. 217.

[6] D.B. Williams and J.I. Goldstein in "Analytical Electron Microscopy 1981”, ed. R.H. Geiss, (San Francisco Press, San Francisco), p. 39.

[7] This work is supported by the Air Force Office of Scientific Research (Grant: FA9550-14-1-0182). The authors also acknowledge the Analytical Instrumentation Facility (AIF) at North Carolina State University, which is supported by the State of North Carolina and the National Science Foundation.
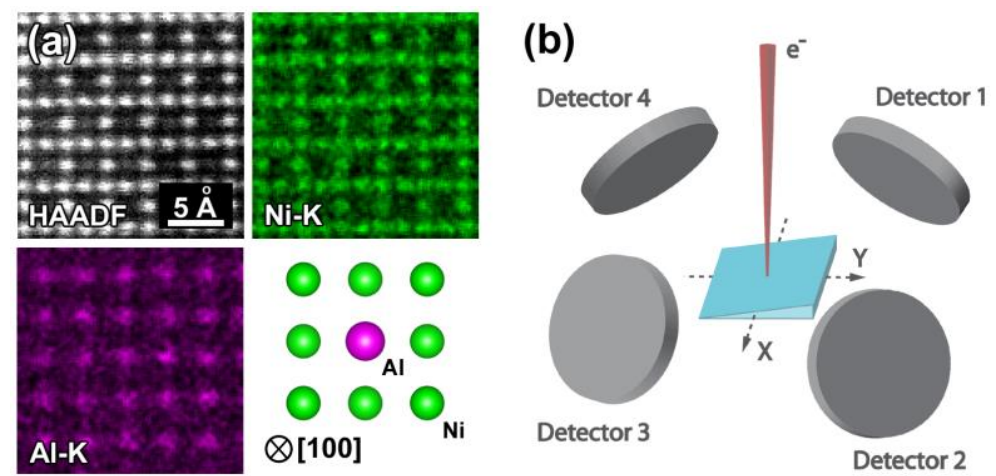

Figure 1. (a) HAADF image and the corresponding atomic-resolution EDS maps (Ni-K and Al-K) for $\mathrm{Ni}_{3} \mathrm{Al}$ oriented along a [100] direction. The EDS maps are filtered using a 3 points average. (b) Schematic illustration of the geometric orientation relationship between the wedge-shape specimen and four-quadrant Super-X detectors.
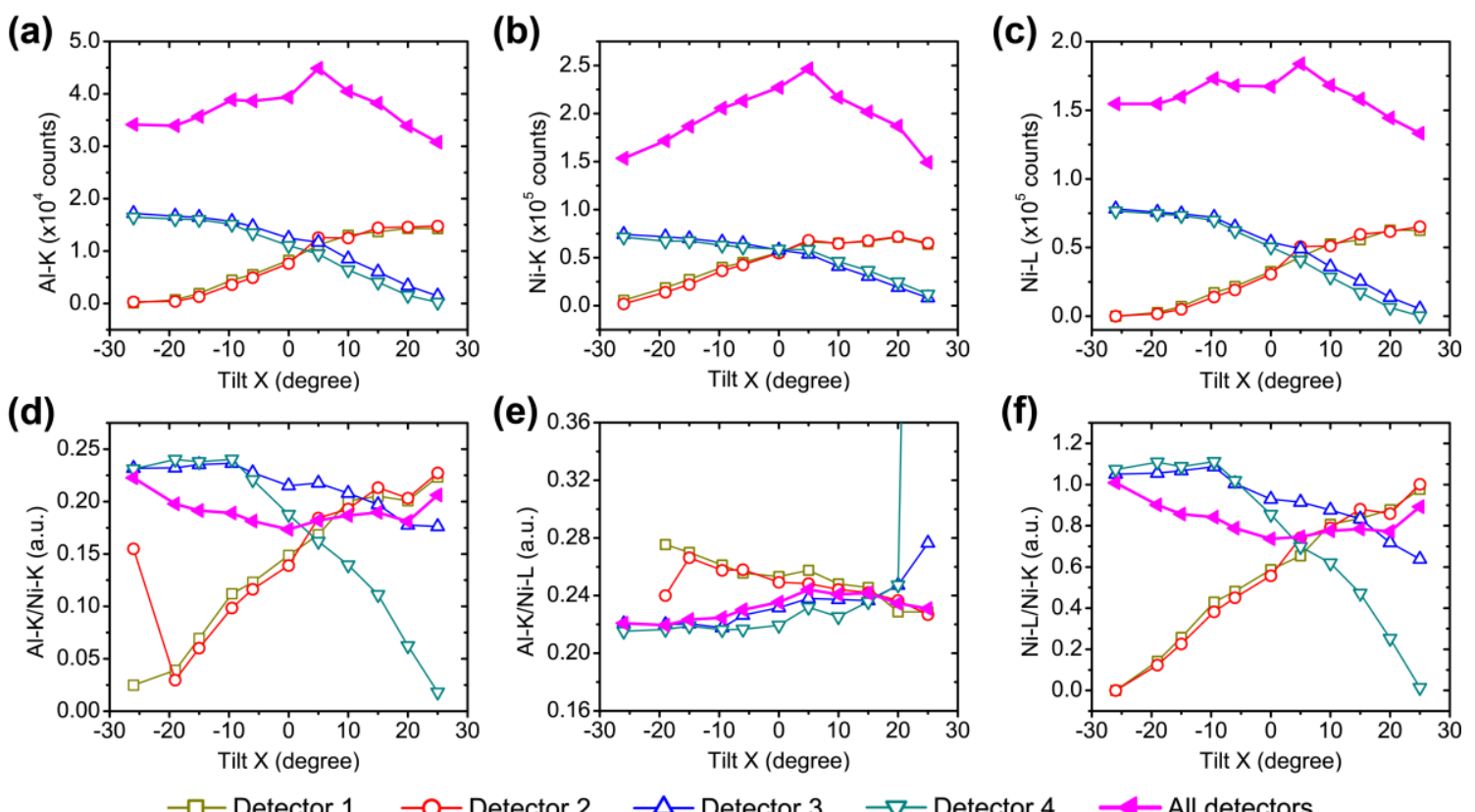

Figure 2. Deviation of X-ray signals from Super-X detectors in terms of (a) Al-K, (b) Ni-L and (c) Ni$\mathrm{K}$ intensities, and their intensity ratio of (d) Al-K/Ni-K, (e) Al-K/Ni-L and (f) Ni-L/Ni-K. Such deviation also varies with the specimen $\mathrm{x}$-tilt, and appears to be non-symmetric. All spectra were obtained from a wedge-polished $\mathrm{Ni}_{3} \mathrm{Al}$ specimen in the same thickness region $(0.5$ mean free path from EELS) and under the same microscope condition (probe-corrected STEM with a 0.11nA and 14 mrad probe). The orientation relationship between the specimen and Super-X detectors is shown in Figure $1 \mathrm{~b}$. 